\title{
Cough syncope and multiple pulmonary nodules
}

\author{
Louis Kreitmann • François Collet • \\ Jean-Paul Gouello $\cdot$ Malcolm Lemyze
}

Received: 27 October 2010/ Accepted: 24 January 2011 / Published online: 13 February 2011

(C) SIMI 2011

A 71-year-old farmer was admitted to the Intensive Care Unit because of acute respiratory failure following a 5-month history of chronic cough. His medical history included a tobacco smoking habit, and severe bilateral carotid stenosis. He had been suffering from exertional dyspnea for many years, but had never sought medical care. He had never travelled abroad, and was taking no medication. The vital signs were; blood pressure $135 / 85 \mathrm{~mm} / \mathrm{Hg}$, pulse 95 beats/min, respiratory rate 24 breaths/min, body temperature $37.3^{\circ} \mathrm{C}$. The physical examination was unremarkable except for bilateral wheezing on lung auscultation.

Arterial blood gas analysis on breathing room air showed pH 7.34, $\mathrm{PCO}_{2} 48 \mathrm{mmHg}, \mathrm{PO}_{2} 56 \mathrm{mmHg}$. Laboratory tests included a slightly elevated $\mathrm{C}$-reactive protein level $46 \mathrm{mg} / \mathrm{L}$, procalcitonin lower than normal $0.05 \mathrm{ng} /$ $\mathrm{mL}$. The blood white-cell count was 10.6 cells $/ \mathrm{mm}^{3}$ with marked hypereosinophilia $\left(2040\right.$ cells $\left./ \mathrm{mm}^{3}\right)$. The chest $\mathrm{X}$-ray study showed no significant abnormality. A thoracic contrast computed tomography (CT scan) ruled out a pulmonary embolism, but showed bilateral nodules and patchy infiltrates, disseminated asymmetrically throughout lung parenchyma (Figs. 1, 2). The patient was initially treated with oxygen supplementation, antibiotics (amoxicilline

L. Kreitmann · M. Lemyze

Department of Respiratory and Critical Care Medicine,

Schaffner Hospital, Lens, France

F. Collet · J.-P. Gouello

Department of Critical Care Medicine,

Broussais Hospital, Saint Malo, France

M. Lemyze $(\bowtie)$

Service de Réanimation polyvalente, Centre Hospitalier Dr. Schaffner, 99 route de la Bassée, 62300 Lens, France e-mail: malcolmlemyze@yahoo.fr clavulanate $1 \mathrm{~g}$ tid) and steroids (prednisone $40 \mathrm{mg} /$ day), resulting in moderate improvement.

Eosinophilia suggested multiple parasitological examinations of the feces; they were all negative. Nevertheless, 6 days after admission, immuno-enzymatic assay (ELISA) for Toxocara Canis appeared strongly positive. It was later confirmed by a western blot analysis. Closer questioning of the patient revealed that he was living alone with his dog after his wife had died 6 months prior. He had given up his daily work on the farm, and progressively had ceased cleaning up the dog's feces. Intermittent dry coughing fits had then appeared, becoming increasingly frequent. Coughing had soon become permanent and disabling with several episodes of cough syncope that had led him to consult his general practitioner.

Diagnosis Visceral larva migrans with pulmonary involvement caused by Toxocara Canis infection.

Visceral larva migrans is a ubiquitous zoonosis resulting from infestation by the common roundworms of dogs and cats, Toxocara Canis and Cati, respectively [1]. The parasite undergoes a complete cycle in young dogs, which leads to the excretion of embryonated eggs in the environment. Human beings are infected after ingestion of the eggs, either via contaminated food, or (more commonly) via unwashed hands. The larvae that hatch from the eggs then invade the intestinal mucosa and disseminate to several organs, including the liver, the central nervous system and the lungs $[1,3]$.

In immunocompetent adult hosts, toxocarosis is most often asymptomatic, but can exhibit a wide variety of clinical features reflecting visceral spread of the larvae [1]. Mild, chronic infections usually presents with the clinical pictures of "covert" or "common" toxocariosis, while the term "visceral larva migrans" reflects more acute multiorgan presentations. Pulmonary involvement is reported in 


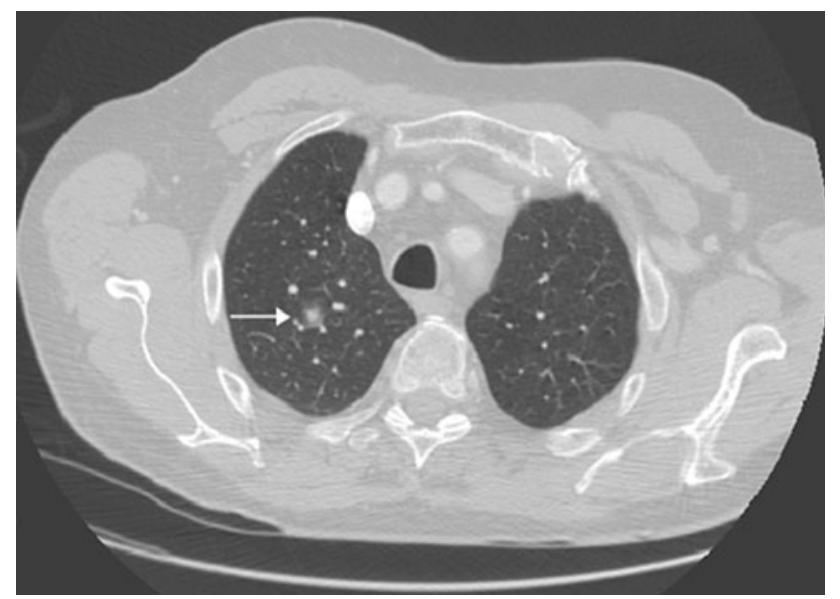

Fig. 1 Chest CT scan showing an isolated pulmonary nodule in the upper lobe of the right lung (arrow)

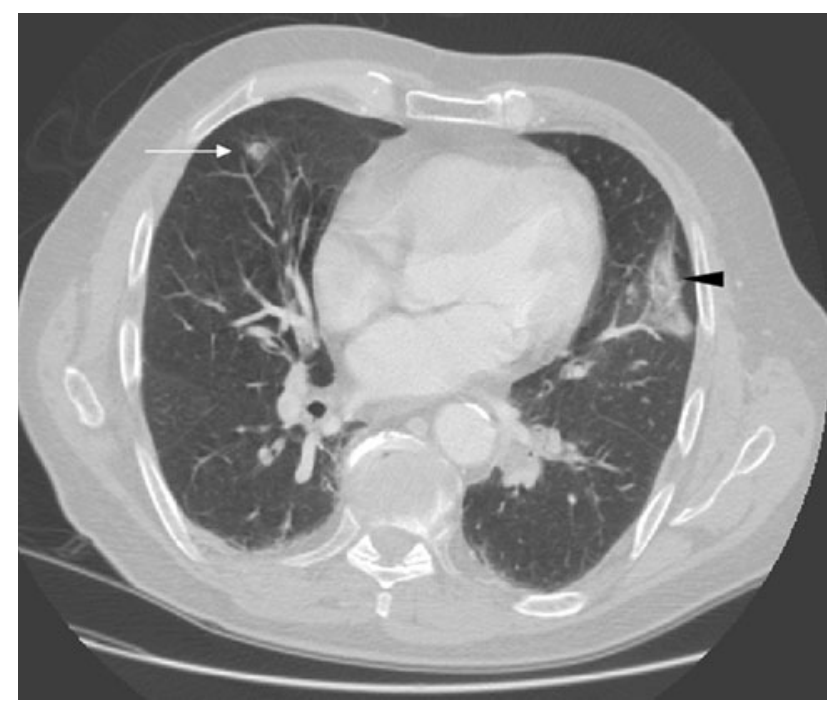

Fig. 2 Chest CT scan showing airspace nodule with surrounding ground-glass opacity in the lower lobe of the right lung (arrow) and a pulmonary infiltrate in the left lower lobe (arrowhead).

approximately $50 \%$ of cases. The most common respiratory symptoms are cough, often with chronic wheezing coughing spells, fever and mild dyspnea [3]. Eosinophilia and elevation of $\operatorname{IgE}$ are nonspecific, but they have good sensitivity and should suggest a possible parasitic etiology [1]. Broncho-alveolar lavage shows marked eosinophilia (usually $>25 \%$ ) [3]. Inflammatory markers such as CRP and PCT levels may be negative, as is parasitological examination of the feces (since there is no fecal excretion of eggs in non-capable hosts such as humans). Chest radiographs and CT scans exhibit mild, patchy, unilateral or bilateral infiltrates associated with pulmonary nodules $[2,5]$.

Our patient presented with cough syncope, a rare manifestation that has not been reported in other cases of visceral larva migrans. In the present case, coughing may have resulted in an increased intra-thoracic pressure, compromising venous return from the superior cave territory and thus leading to increased intra-cerebral pressure. Cardiac output and the brain's oxygen supply are usually reduced in patients with severe bilateral carotid stenosis. The combination of both mechanisms may have compromised brain perfusion and caused a loss of consciousness.

When confronted with respiratory symptoms associated with eosinophilia, the differential diagnosis includes parasitic infections, drug reactions, allergic manifestations, asthma, pulmonary vasculitis (e.g. Churg and Strauss syndrome), and a wide group of eosinophilic lung diseases of unknown origin [2]. The diagnosis of visceral larva migrans due to Toxocara Canis must hinge upon a positive result of the ELISA test to TES-antigen exhibited by the migrating larvae. This technique is reported to have a sensitivity of $78 \%$ and a specificity of $90 \%$. Several techniques might then be useful to confirm that the infection is recent, such as assay for specific IgE or western blot [1].

To date, there are no guidelines available regarding the treatment of toxocariasis. Several anti-parasitic treatments are effective to eradicate the larvae; we might suggest a treatment with albendazole $10 \mathrm{mg} / \mathrm{kg} /$ day for at least 5 days. Noteworthy, in all patients presenting with either neurologic or ocular involvement, anti-helminthic treatment should be postponed until a 4 to 6 -week course of high-dose corticosteroids (prednisolone $1.5 \mathrm{mg} / \mathrm{kg} /$ day) has been completed [1]. All patients should be carefully advised and informed of specific measures to prevent reinfection, including careful hand washing and food care along with eradication treatment of dogs and cats.

It is thought that up to $50 \%$ of dogs may carry the worm in developed countries, and several studies reveal a seroprevalence in human adults ranging from 4 to $14 \%$ [1, 4], with even higher rates in certain groups (rural or economically disadvantaged populations...). These figures strikingly contrast with the relatively low occurrence of visceral larva migrans, a discrepancy for which several explanations may be hypothesized. First it is possible that toxocariasis may be underdiagnosed because of its nonspecific clinical manifestations [1]. Second, patients presenting with pulmonary involvement are likely to receive steroids in the course of their treatment, which may eradicate the eosinophilia, and thus deprive the physician of the main biological hint to the diagnosis. Finally, we believe the clinical course of toxocariasis infection to reflect complex interactions between the worm and its host. It is indeed possible that severe disease is seen only in specific high-risk populations such as immunocompromised or respiratory-disabled patients. The molecular mechanisms leading from soil contamination to active disease are poorly understood, but further research will certainly 
provide new insight into the determinants of the clinical expression of toxocariasis.

The Internal and Emergency Medicine consent form has been signed by the patient.

\section{Conflict of interest None.}

\section{References}

1. Magnaval JF et al (2001) Highlights of human toxocariasis. Korean J Parasitol 39(1):1-11
2. Jeong YJ, Kim KI, Seo IJ, Lee CH, Lee KN, Kim KN, Kim JS, Kwon WJ (2007) Eosinophilic lung diseases: a clinical, radiologic, and pathologic overview. Radiographics 27:617-637

3. Inoue $\mathrm{K}$, Inoue $\mathrm{Y}$, Arai T, Nawa Y, Kashiwa Y, Yamamoto S, Sakatani M (2002) Chronic eosinophilic pneumonia due to visceral larva migrans. Intern Med 41:478-482

4. Won KY, Kruszon-Moran D, Schantz PM, Jones JL (2008) National seroprevalence and risk factors for Zoonotic Toxocara spp. infection. Am J Trop Med Hyg 79(4):552-557

5. Sakai S, Shida Y, Takahashi N, Yabuuchi H, Soeda H, Okafuji T, Hatakenaka M, Honda H (2006) Pulmonary lesions associated with visceral larva migrans due to Ascaris suum or Toxocara canis: imaging of six cases. AJR Am J Roentgenol 186:1697-1702 